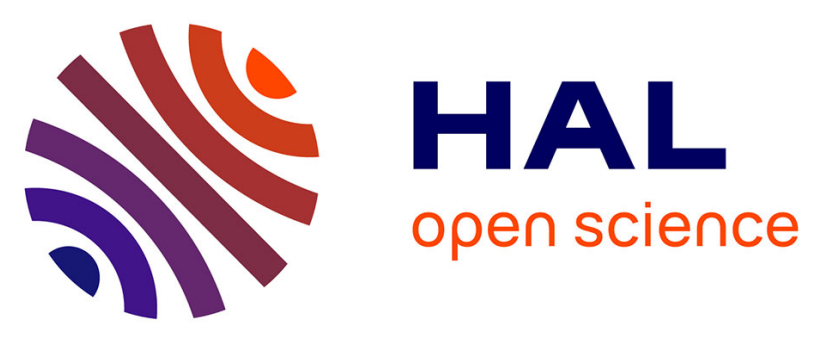

\title{
Impact of spectral phase mismatch on femtosecond coherent beam combining systems
}

Louis Daniault, Marc Hanna, Laurent Lombard, Yoann Zaouter, Eric Mottay, Didier Goular, Pierre Bourdon, Frédéric Druon, Patrick Georges

\section{- To cite this version:}

Louis Daniault, Marc Hanna, Laurent Lombard, Yoann Zaouter, Eric Mottay, et al.. Impact of spectral phase mismatch on femtosecond coherent beam combining systems. Optics Letters, 2012, 37 (4), pp.650-652. hal-00702163

\section{HAL Id: hal-00702163 \\ https://hal-iogs.archives-ouvertes.fr/hal-00702163}

Submitted on 29 May 2012

HAL is a multi-disciplinary open access archive for the deposit and dissemination of scientific research documents, whether they are published or not. The documents may come from teaching and research institutions in France or abroad, or from public or private research centers.
L'archive ouverte pluridisciplinaire HAL, est destinée au dépôt et à la diffusion de documents scientifiques de niveau recherche, publiés ou non, émanant des établissements d'enseignement et de recherche français ou étrangers, des laboratoires publics ou privés. 


\title{
Impact of spectral phase mismatch on femtosecond coherent beam combining systems
}

\author{
L. Daniault ${ }^{1}$, M. Hanna ${ }^{1}$, L. Lombard ${ }^{2}$, Y. Zaouter ${ }^{3}$, E. Mottay ${ }^{3}$, D. Goular ${ }^{2}$, P. Bourdon ${ }^{2}$, F. Druon \\ and P. Georges ${ }^{1}$ \\ 1. Laboratoire Charles Fabry de l'Institut d'Optique, UMR 8501 CNRS, Université Paris Sud, 91127 Palaiseau, France \\ 2. Office National d'Etudes et de Recherches Aérospatiales, DOTA, Chemin de la Hunière, 91761 Palaiseau, France \\ 3. Amplitude Systèmes, 11 avenue de Canteranne, Cité de la Photonique, 33600 Pessac, France \\ *Corresponding author: louis.daniault@institutoptique.fr
}

Received Month X, XXXX; revised Month X, XXXX; accepted Month X, XXXX; posted Month X, XXXX (Doc. ID XXXXX); published Month X, XXXX

\begin{abstract}
We experimentally investigate the impact of spectral phase mismatch on the coherent beam combining of two femtosecond fiber chirped-pulse amplifiers. By measuring the differential spectral phase, both linear and nonlinear contributions are identified. An accumulated nonlinear phase as high as 6 rad has been measured, for which a combination efficiency of $91 \%$ can be obtained by symmetrizing the pump and injection powers. This also allows us to quantitatively separate the spatial and temporal contributions of the non-perfect combining efficiency.

OCIS Codes: $060.2320,140.3298,140.7090$
\end{abstract}

In ultrafast ytterbium-doped fiber amplifiers, the onset of nonlinear effects, especially self-phase modulation (SPM), implies limited pulse energies. One way to overcome this drawback is to use large mode area (LMA) fibers that are less subject to SPM effects at equivalent output powers. This core size scaling is limited by the appearance of higher-order transverse modes, thereby limiting the scalability. A solution to reach higher energies consists in coherently combining the outputs of several fiber amplifiers in free space. This approach has recently been demonstrated in femtosecond regime with two fiber amplifiers, both in active [1-3] and passive [4] architectures.

In contrast with $\mathrm{CW}$ [5] and ns [6] regimes, the efficient combination of femtosecond pulses requires the optical phase to be matched over the whole spectrum: in addition to the zero-order phase stabilization, the shape of the spectral phase must be identical in both arms. In the linear amplification regime (negligible B-integrals) and for pulses longer than $100 \mathrm{fs}$, this essentially means that the two first orders of the spectral phase, i.e. group delay and group-delay dispersion, should be identical. Experiments showed that a static adjustment of these quantities is sufficient to obtain good combination over hours [2] and high efficiencies up to $97 \%$ have been demonstrated [1] However, all practical high-energy femtosecond fiber systems operate at non negligible B-integrals. In these nonlinear amplification regimes, the accumulation of SPM results in a distortion of the spectral phase profile. It is therefore important to investigate the compatibility of the nonlinear regime with coherent beam combining.

In this Letter, we experimentally investigate the influence of dispersion and SPM effects on the coherent beam combining of two femtosecond fiber chirped-pulse amplifiers. Although coherent combining experiments with non-negligible B-integrals have already been demonstrated [2,4], no detailed analysis on dispersion and nonlinear phase management has yet been presented. In this Letter, a way to identify and quantify the various contributions to the non-perfect combining efficiency is described. The measurement of the relative spectral phase between both arms of the combining system allows us to precisely balance the SPM effects, resulting in a combining efficiency of $91 \%$ at a B-integral of $6 \mathrm{rad}$ in each amplifier. This method also allows us to determine the spectral and spatial contributions separately.

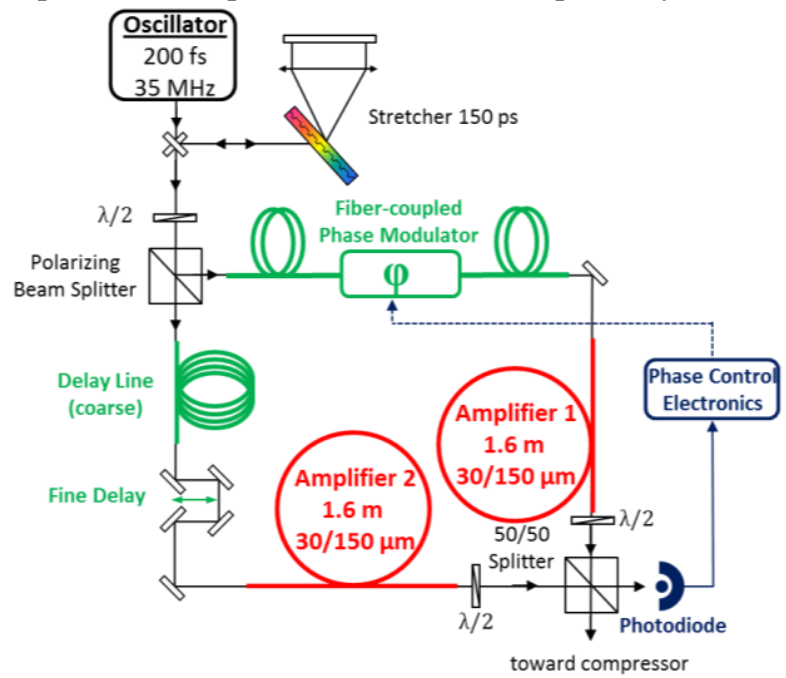

Fig.1 (Color online): Experimental setup

The experimental setup is described in Fig. 1. A femtosecond $\mathrm{Yb}^{3+}: \mathrm{KYW}$ oscillator delivering 200 fs pulses at $1030 \mathrm{~nm}$ and $35 \mathrm{MHz}$ repetition rate is followed by an acousto-optic modulator and a stretcher that broadens the pulses to $100 \mathrm{ps}$. A half-wave plate with a polarizing beam splitter is used to divide the seed into two arms with adjustable input powers. The first arm consists of a fibercoupled $\mathrm{LiNbO}_{3}$ integrated phase modulator followed by a 
$1.6 \mathrm{~m}$ Yb-doped 30/150 $\mu \mathrm{m}$ large mode area (LMA) fiber amplifier. The second arm contains a $2.4 \mathrm{~m}$ single-mode fiber, a free-space delay line, and another $1.6 \mathrm{~m}$ of the same LMA fiber. The single-mode fiber is used to match the group-velocity dispersion of both arms, and also acts as a coarse delay matching element. Both amplifiers are seeded with $50 \mathrm{~mW}$ average power at $35 \mathrm{MHz}$ and are pumped to deliver identical powers. The outputs of the fiber amplifiers are collimated and overlap on a 50/50 beam splitter. The constructive output of the beam splitter goes to the compressor that exhibits $65 \%$ efficiency. The rejected output is followed by a photodiode that detects an error signal to be minimized. The zeroorder active phase control system is the same as described in [3], with an improved bandwidth of $10 \mathrm{kHz}$.

By changing the reference phase of the lock-in amplifier in the phase control electronics by $\pi$, we are able to switch the 50/50 beam splitter outputs, so that both the combined and rejected beams can be characterized. At the output, the spectra of channels 1 and 2, along with the combined and the rejected beams are measured. The spectrum at the output is given by the standard interference expression for each frequency component,

$$
I(\omega)=I_{1}(\omega)+I_{2}(\omega)+2 \sqrt{I_{1}(\omega) I_{2}(\omega)} \cos (\varphi(\omega),
$$

allowing us to retrieve the absolute value of the relative spectral phase $\Delta \varphi(\omega)$ between the two incident beams. As opposed to single pulse complete characterization techniques such as frequency-resolved optical gating, this method is differential and includes zeroth- and first-order contributions of the relative spectral phase. It is therefore well-suited to measuring precisely spectral phase mismatch.

For reference, the system is first operated in linear regime at $35 \mathrm{MHz}$ repetition rate. A combined output power of $12.5 \mathrm{~W}$ is measured before compression, corresponding to $92 \%$ combining efficiency and stable for several hours. The autocorrelation of the recombined pulse is shown in Fig. 2, and corresponds to a $230 \mathrm{fs}$ pulsewidth assuming a sech-square shape, a record value for a beam combining experiment.
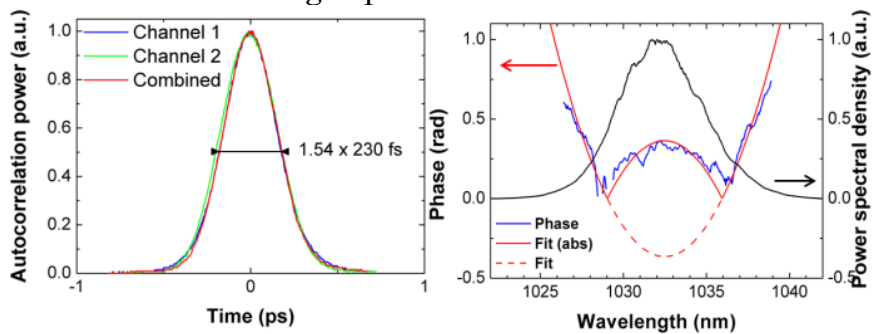

Fig.2 (Color online): Autocorrelation of channels 1, 2 and combined beam (left); Spectrum of the combined beam and relative spectral phase between the two initial channels (right)

The absolute value of the relative phase is retrieved using the method described above, and is plotted in Fig. 2 along with the combined spectrum. The curve is fitted with the absolute value of a parabola $\varphi(\omega)=\varphi_{0}+\varphi_{1} \omega+\varphi_{2} \omega^{2}$ that takes into account the zeroorder phase, delay and group-velocity dispersion discrepancies respectively. In this expression, the zero frequency is set to be the mean frequency of the pulse. The fit coefficients exhibit a zero-order term $\varphi_{0}=-0.3 \mathrm{rad}$, and a delay of $20 \mathrm{fs}$ between the pulses using $\varphi_{1}$. The second-order term, which mostly contributes to the efficiency losses, allows an estimation of the pulse duration discrepancy, but cannot be solely attributed to fiber length mismatch. Indeed, the mirrors in the setup are not GVD controlled, and introduce random amounts of second-order phase. Since the phase modulator tracks the maximum efficiency, the zero-order term $\varphi_{0}$ is not null and set to partially compensate the high-order contributions. To control the validity of these measurements, we reconstruct the recombined spectrum using the experimental spectra of the two channels and the fitted spectral phase. The result is in excellent agreement with the experiment and corresponds to a combining efficiency of $97 \%$. This implies that $3 \%$ of the overall combining losses are due to spectral effects, while the remaining losses are related to polarization and spatial issues, such as wavefront and beam profile discrepancies and estimated here to 5\%. The combining efficiency is also calculated with $\varphi_{0}$ varying from 0 to 2 , which simulates the phase modulator tracking. Its curve exhibits a cosine shape with a maximum at $97 \%$ and a corresponding $\varphi_{0}=-0.2 \mathrm{rad}$, in good agreement with the measured value for $\varphi$.

To investigate the nonlinear regime, the repetition rate is set to $1 \mathrm{MHz}$. In that case, each channel experiences a priori the same SPM effects, which should not affect the combining efficiency. In practice, the accumulated nonlinear phase is different, and leads to a significant drop of the combining efficiency. The spectral phase measurement provides a way to measure and compensate for this effect. For moderate SPM effects, the nonlinear contribution in the spectral phase can be expressed as proportional to the spectral shape [7]. With a spectrum shape normalized to 1 , the related coefficient is directly the B-integral of the pulse. Considering similar spectra for the incoming pulses, as it is almost the case experimentally, the nonlinear contribution to the relative spectral phase corresponds to the B-integral discrepancy between the two channels, weighted by the initial spectra.

To take into account the nonlinear contribution to the differential spectral phase, the fit expression is modified as

$$
\varphi(\omega)=\varphi_{0}+\varphi_{1} \omega+\varphi_{2} \omega^{2}+\Delta B I_{N}(\omega),
$$

with $\Delta \mathrm{B}$ the B-integral discrepancy and $\mathrm{I}_{\mathrm{N}}(\omega)$ the normalized fit of one of the two incoming spectra.

The study in nonlinear regime is performed in two configurations. The first series of measurements is realized by adjusting the pump powers so that both arms deliver the same output power. The corresponding output 
power is measured to be $8.8 \mathrm{~W}$ and exhibits $86 \%$ combining efficiency, illustrating the nonlinearitiesrelated efficiency drop. The autocorrelation of the two initial and the recombined pulses are shown in Fig. 3 (left). The B-integral experienced by each pulse is estimated around $6 \mathrm{rad}$. The two incoming pulses do not exhibit the same temporal shape and have experimentally different compression points, although they present similar shapes at best compression. The spectrum of the combined pulses and its associated relative spectral phase are plotted in Fig. 3 (left). The B-integral discrepancy derived from the phase fit is $\Delta \mathrm{B}=2.5 \mathrm{rad}$ and the zeroorder phase is now $\varphi_{0}=-2 \mathrm{rad}$. The origin of this high value of B-integral discrepancy, despite similar output powers, is not well identified. Possible reasons include differences in injection conditions and polarizations at the input and fiber parameter discrepancy. The retrieved $\varphi_{2}$ value is unchanged from the linear case. The delay value is $60 \mathrm{fs}$, higher than the $20 \mathrm{fs}$ in linear regime. This additional contribution comes from dissymmetry in the combined spectrum that introduces odd-order terms, and can be partially compensated in the setup using the fine delay. The combining efficiency calculated from the spectral data is $90 \%$, implying here $4 \%$ of spatial overlap related losses.
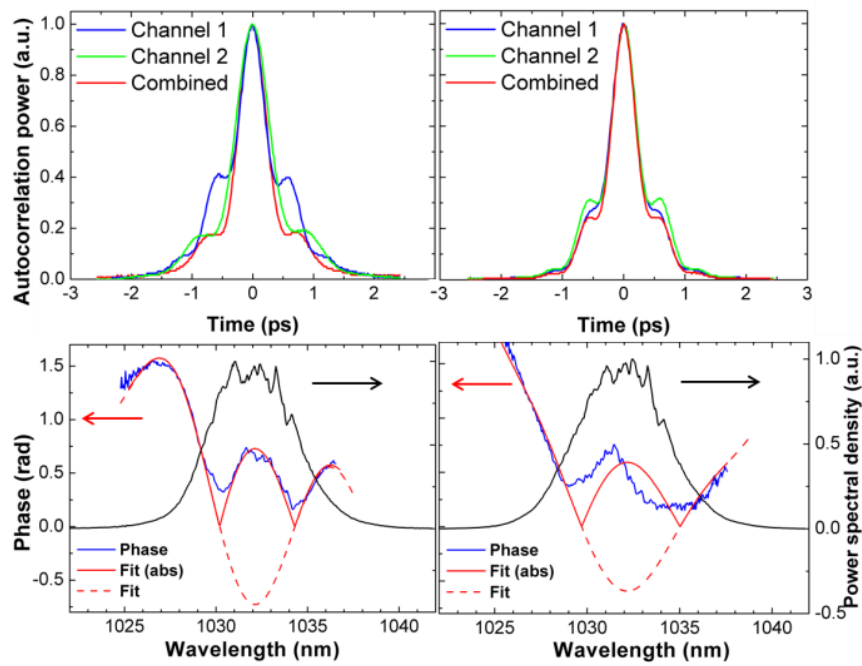

Fig.3 (Color online): Autocorrelation of channels 1, 2 and combined beam (top); Spectrum of the combined beam and relative spectral phase between the two initial channels (bottom); Left: unmatched case; Right: balanced SPM effects.

In the second configuration, the nonlinear effects are balanced by tuning the injection and pump powers [8] together with the delay, in order to minimize the rejected power. This is achieved by observing the behavior of the uncombined spectral content and minimizing it carefully. In this situation, the two amplifiers do not deliver the same power and the related efficiency losses are $0.5 \%$. This new configuration exhibits a combining efficiency of $91 \%$ with $9.2 \mathrm{~W}$ output power, i.e. only $1 \%$ less than in linear regime. The autocorrelations of each channel and the combined beam are shown in Fig. 3 (right). The incoming and combined pulses now share the same shape and compression point. The autocorrelation FWHM is 410 fs, yielding pulse duration of 320 fs according to simulations. The combined spectra are plotted with the relative spectral phase in Fig.3. Noticeable differences appear between the fit and the experimental data in the middle of the spectrum. This comes from differences between the arms in power (ratio $=0.7$ ) and central wavelength $(\Delta \lambda=1 \mathrm{~nm})$ that appear due to the injection condition modifications. The recovered B-integral difference decreases to $\Delta \mathrm{B}=-0.4 \mathrm{rad}$ and the delay to 25 fs. The second-order term still matches the linear regime and thus introduces the same losses. The efficiency calculated from the spectral data is $96 \%$, showing that the spatial overlap related losses still remain around 5\%. Thus, the measurement allows us to improve the system efficiency by balancing the B-integrals in both arms. This operation is gainful even in the present case where it results in slightly imbalanced output power, because the efficiency is much more impacted by phase mismatch than by intensity mismatch.

In conclusion, we demonstrate the coherent beam combining of two fiber chirped-pulse amplifiers with a high-accumulated nonlinear phase of $6 \mathrm{rad}$ and show that the efficiency demonstrated in linear regime can be maintained. The measurement of the relative spectral phase provides a powerful tool to analyze different contributions to the non-ideal combining efficiency This analysis and identification provide guidelines to experimentally optimize coherent combining systems, even in the case of stretcher-free highly nonlinear systems systems delivering sub $100 \mathrm{fs}$ pulses.

The research described here has been supported by Triangle de la Physique contract 2009-026T, and Agence Nationale de la Recherche through the Multifemto project.

\section{References}

1. E. Seise, A. Klenke, J. Limpert, and A. Tünnermann, Opt. Express 18, 27827-27835 (2010).

2. E. Seise, A. Klenke, S. Breitkopf, J. Limpert, and A. Tünnermann, Opt. Lett. 36, 3858-3860 (2011)

3. L. Daniault, M. Hanna, L. Lombard, Y. Zaouter, E. Mottay, D. Goular, P. Bourdon, F. Druon, and P. Georges, Opt. Lett. 36, 621-623 (2011).

4. L. Daniault, M. Hanna, D. N. Papadopoulos, Y. Zaouter, E. Mottay, F. Druon, P. Georges, Opt. Lett., 36, XXXX (2011).

5. S. J. Augst, J. K. Ranka, T. Y. Fan, and A. Sanchez, J. Opt. Soc. Am. B 24, 1707-1715 (2007)

6. L. Lombard, A. Azarian, K. Cadoret, P. Bourdon, D. Goular, G. Canat, V. Jolivet, Y. Jaouën, and O. Vasseur, Opt. Lett. 36, 523-525 (2011).

7. A. Galvanauskas, Ultrafast Lasers (CRC, 2002), p. 209.

8. S. Jiang, M. Hanna, F. Druon, and P. Georges, Opt. Lett. 35,1293 (2010). 
1. Enrico Seise, Arno Klenke, Jens Limpert, and Andreas Tünnermann, "Coherent addition of fiber-amplified ultrashort laser pulses," Opt. Express 18, 27827-27835 (2010)

2. Enrico Seise, Arno Klenke, Sven Breitkopf, Jens Limpert, and Andreas Tünnermann, "88 W 0.5 mJ femtosecond laser pulses from two coherently combined fiber amplifiers," Opt. Lett. 36, 3858-3860 (2011)

3. L. Daniault, M. Hanna, L. Lombard, Y. Zaouter, E. Mottay, D. Goular, P. Bourdon, F. Druon, and P. Georges, "Coherent beam combining of two femtosecond fiber chirped-pulse amplifiers," Opt. Lett. 36, 621-623 (2011)

4. Louis Daniault, Marc Hanna, Dimitris N. Papadopoulos, Yoann Zaouter, Eric Mottay, Frédéric Druon, and Patrick Georges, "Passive coherent beam combining of two femtosecond fiber chirped-pulse amplifiers," Opt. Lett. 36, 4023-4025 (2011)

5. Steven J. Augst, Jinendra K. Ranka, T. Y. Fan, and Antonio Sanchez, "Beam combining of ytterbium fiber amplifiers (Invited)," J. Opt. Soc. Am. B 24, 1707-1715 (2007)

6. L. Lombard, A. Azarian, K. Cadoret, P. Bourdon, D. Goular, G. Canat, V. Jolivet, Y. Jaouën, and O. Vasseur, "Coherent beam combination of narrow-linewidth 1.5um fiber amplifiers in a long-pulse regime," Opt. Lett. 36, 523-525 (2011)

7. Almantas Galvanauskas, Ultrafast Lasers (CRC, 2002), p. 209.

8. Shifeng Jiang, Marc Hanna, Frédéric Druon, and Patrick Georges, "Impact of self-phase modulation on coherently combined fiber chirped-pulse amplifiers," Opt. Lett. 35, 1293-1295 (2010) 